\title{
Inhibitory effects of epigenetic modulators and differentiation inducers on human medulloblastoma cell lines
}

\author{
Ina Patties*, Rolf-Dieter Kortmann and Annegret Glasow
}

\begin{abstract}
Background: Medulloblastoma (MB) is the most common malignant brain tumor in childhood with a 5-year survival of approximately $60 \%$. We have recently shown that treatment of human MB cells with 5-aza-2'-deoxycytidine (5-aza-dC) reduces the clonogenic survival significantly. Here, we tested combinatorial effects of 5 -aza-dC with other epigenetic (valproic acid, SAHA) and differentiation-inducing drugs (resveratrol, abacavir, retinoic acid) on human MB cells in vitro to intensify the antitumor therapy further.

Methods: Three human MB cell lines were treated with 5-aza-dC alone or in combination for three or six days. Metabolic activity was measured by WST-1 assay. To determine long-term reproductive survival, clonogenic assays were performed. Induction of DNA double-strand break (DSB) repair was measured by $\gamma \mathrm{H} 2 \mathrm{AX}$ assay.

Results: The applied single drugs, except for ATRA, reduced the metabolic activity dose-dependently in all MB cell lines. Longer treatment times enhanced the reduction of metabolic activity by 5 -aza-dC. Combinatorial treatments showed differential, cell line-dependent responses indicating an important impact of the genetic background. 5-Aza-dC together with resveratrol was found to exert the most significant inhibitory effects on metabolic activity in all cell lines. 5-aza-dC alone reduced the clonogenicity of MB cells significantly and induced DSB with no further changes after adjuvant administration of resveratrol.

Conclusion: The observed significant decrease in metabolic activity by combinatorial treatment of MB cells with 5-aza-dC and resveratrol does not translate into long-term reproductive survival deficiency in vitro. Further studies in animal models are needed to clarify the resveratrol-mediated anticancer mechanisms in vivo.
\end{abstract}

Keywords: Medulloblastoma, 5-Aza-2'-deoxycytidine, Resveratrol, Abacavir, Valproic acid, Retinoic acid, SAHA

\section{Background}

Medulloblastoma (MB) is the most common malignant brain tumor in childhood and accounts for $20 \%$ of such entities. It arises during embryonic development from neural precursor cells in the precerebellum or the dorsal brain stem [1]. It is widely believed that genetic, gene regulatory, or epigenetic abnormalities give rise to tumor initiation and inhibit normal neuronal or glial differentiation in neural stem cells [2]. MB standard therapy includes primary tumor resection followed by irradiation and/or chemotherapy. At the moment, therapy stratification depends on tumor histology, metastasis stage, and

\footnotetext{
* Correspondence: Ina.patties@medizin.uni-leipzig.de

Department of Radiation Therapy, University of Leipzig, Stephanstraße 9a, Leipzig 04103, Germany
}

patient age. Patients belonging to the high-risk group and such with metastases receive a more intensive concomitant chemoradiotherapy compared to low-risk patients. Infants below 18 months do not obtain radiation therapy to avoid radiation-related adverse late effects, like neurocognitive and psychomotoric deficits, but receive a highly aggressive chemotherapy. With overall 5 -year survival rates of approximately $60 \%$, an improved antitumor strategy is urgently needed to further enhance the outcome of the moderate- and high-risk patients ( $90 \%$ of all MB patients). Especially in younger children, a reduction of treatment-induced adverse effects, by applying less toxic agents, is an ambitious aim in $\mathrm{MB}$ therapy optimization. 
Epigenetic aberrations like HIC1, RASSF1a, or CASP8 promoter methylation, which are observed in most MBs (70-90\%), lead to silenced tumor suppressor genes (TSG) and are responsible for the lack of cell cycle arrest and apoptosis in tumor cells [2]. Hence, the application of epigenetic modulators in the treatment of $\mathrm{MB}$ might be a suitable approach to improve the standard therapy. Methyltransferase inhibitors like 5-aza-2'-deoxycytidine (5-aza-dC, decitabine) and histone deacetylase inhibitors (HDACi) like valproic acid (VPA) or SAHA are approved for the therapy of other diseases such as myelodysplastic syndromes, neurological disorders, or T-cell lymphoma. Application of epigenetic drugs in leukemia and carcinomas is currently tested in clinical studies. In addition, the low differentiation stage of $\mathrm{MB}$ cells constitutes also an attractive approach for MB therapy. The usage of differentiation-inducing drugs may induce neuronal or glial maturation in tumor cells and, therefore, eliminate their cancer-causing abilities. For instance, all-trans retinoic acid (ATRA) has already been used in differentiation therapy of leukemia patients. In vitro experiments with abacavir and resveratrol exhibited the drug-mediated induction of a more differentiated cell phenotype in $\mathrm{MB}$ cell lines [3-5]. Combination of nucleoside analogs like 5 -aza-dC with $\mathrm{HDACi}$ might result in amplified effects as HDACi have been shown to suppress the alien nucleotide removal [6]. Also, induction of differentiation might work much more successfully after reactivation of beforehand silenced differentiationrelevant genes [7].

In this study, we tested single and combinatorial effects of 5-aza-dC with other epigenetic drugs (VPA, SAHA) or differentiation inducers (resveratrol, abacavir, ATRA), as detailed below, on the metabolic activity and reproductive survival of human $\mathrm{MB}$ cell lines.

5-Aza-dC, a cytidine analog and methyltransferase inhibitor, is approved by the U.S. Food and Drug Administration (FDA) for the treatment of myelodysplastic syndrome since 2006. 5-Aza-dC is known to reactivate silenced TSG by demethylation of their promoter regions in $\mathrm{MB}$ and other tumor cells after incorporation into the DNA during the replication process [8-10]. DNA-integrated 5-aza-dC traps de novo methyltransferases (DNMT) and induces DNA damage including double-strand breaks (DSB) $[11,12]$. We have recently shown that 5 -aza-dC treatment of human $\mathrm{MB}$ cells reduces their vitality, proliferation rate, and clonogenic survival significantly [8]. Others have described similar effects in leukemia and lung cancer cell lines $[13,14]$.

VPA, an HDACi, has already been established in the treatment of epilepsy and depression, and clinical trials for its application in HIV and cancer patients are ongoing. VPA leads to hyperacetylation of histone proteins resulting in activation of cell cycle arrest and apoptosis in human MB cells [15]. In xenograft MB mouse models, it was shown that VPA alone reduces tumor growth and prolonges survival [16]. It was also reported that combinatorial treatment with 5-aza-dC and VPA is able to diminish tumor initiation in a Ptch-deficient MB mouse model [17].

SAHA (vorinostat, Zolinza ${ }^{\mathrm{Tm}}$ ) is the first HDACi approved by the FDA for cancer treatment. SAHA directly interacts with the catalytic domain of histone deacetylases [18]. As a result, gene promoter-bound histones stay hyperacetylated and facilitate the selective transcription of genes [19]. Additionally, SAHA exerts chemosensitizing effects in oral squamous cell carcinoma and medulloblastoma cells $[20,21]$.

Abacavir, a 2-deoxyguanine analog, is approved for HIV and AIDS therapy in the EU since 1999. Two ways of an abacavir-mediated reduction of telomerase activity are reported: 1 ) indirect, by incorporation into the DNA strand which leads to polymerization stop [22], and 2) direct, by downregulation of hTERT (human gene for telomerase reverse transcriptase) mRNA transcription [3]. In recent years, abacavir attracted attention for cancer therapy for its ability to inhibit telomerase activity, which is known to be overexpressed in the vast majority of cancers [23]. Also in 70\% of MBs, telomerase activity is enhanced in contrast to normal cerebellum [24]. It was previously shown that treatment of human MB cell lines with abacavir results in proliferation inhibition and neuronal differentiation [3].

ATRA is the prototype of differentiation therapy in cancer cells and, therefore, it is approved for treatment of acute promyelocytic leukemia (APL) in the EU since 1996. Inhibition of proliferation and induction of apoptosis and differentiation have been observed in many tumor cells including $\mathrm{MB}$ cells after treatment with ATRA [25-30].

Resveratrol, a plant polyphenol, is described to exhibit tumor-preventive as well as anticancer effects dependent on concentration, cell type, and microenvironment [31-33]. In MB cells, resveratrol has been shown to induce cell cycle arrest, apoptosis, and/or neuronal differentiation $[4,5,34]$.

In this study, we show that the applied single mediators, except for ATRA, reduce the metabolic activity in all $\mathrm{MB}$ cell lines. In combinatorial treatments with the epigenetic modifier 5 -aza-dC, resveratrol reveals the strongest decrease in metabolic activity, but it can not further reduce the 5-aza-dC-induced decrease of clonogenic survival.

\section{Methods}

\section{Modulators}

5-Aza-2'deoxycytidine (decitabine, trade name Dacogen ${ }^{\circledR}$ ), all-trans retinoic acid (ATRA), resveratrol, and valproic acid were purchased from Sigma-Aldrich (Munich, 
Germany). Abacavir hemisulfate was kindly provided from GlaxoSmithKline (Hamburg, Germany) and suberoylanilide hydroxamic acid (SAHA, vorinostat, trade name Zolinza $^{\circledR}$ ) from MSD (Haar, Germany). Stock solutions were prepared as follows and stored at $-20^{\circ} \mathrm{C}: 10 \mathrm{mM}$ 5-aza-dC in PBS; $500 \mu \mathrm{M}$ ATRA in 10\% ethanol (stored at $-80^{\circ} \mathrm{C}$ ); $500 \mu \mathrm{M}$ resveratrol in $1 \%$ ethanol; $1 \mathrm{M}$ valproic acid in PBS; $100 \mathrm{mM}$ abacavir in PBS; $100 \mu \mathrm{M}$ SAHA in $0.25 \%$ DMSO. Further work solutions were made in PBS and administered in equal dilutions to the cell medium. To exclude effects based on ethanol or DMSO applications, appropriate controls were implemented.

\section{Cell lines and cell culture}

The human MB cell line MEB-Med8a was kindly provided by Prof. T. Pietsch (Department of Neuropathology, University of Bonn Medical Centre, Bonn, Germany). The MB cell lines D283-Med and DAOY were purchased from ATCC cell biology collection (Manassas VA, USA). D283Med and DAOY were maintained in MEM (SigmaAldrich, Munich, Germany) including $2 \mathrm{mM}$ L-glutamine (Biochrom, Berlin, Germany), MEB-Med8a in DMEM with 4.5 g glucose (Lonza, Basel, Switzerland), all supplemented with 10\% FCS (PAA, Yeovil, Somerset, UK), $100 \mathrm{U} / \mathrm{ml}$ penicillin, and $100 \mu \mathrm{g} / \mathrm{ml}$ streptomycin (Biochrom, Berlin, Germany) at $37^{\circ} \mathrm{C}$ and $5 \% \mathrm{CO}_{2}$ unless otherwise noted.

\section{Metabolic activity}

To examine metabolic activity, cells were seeded in triplicates in 96-well plates, and after $24 \mathrm{~h}$ cells were grown with or without the modulator for three or, in case of 5 -aza-dC, for three and six days. Combinatorial treatments were executed with/without $3 \mu \mathrm{M}$ (D283-Med) or $5 \mu \mathrm{M}$ (DAOY, MEB-Med8a) 5-aza-dC and the second drug (concentrations listed in Table 1). After incubation, medium was discarded, and cells were incubated with normal medium including 10\% WST-1 reagent (Roche, Basel, Switzerland) for 1-2 h. Metabolically active cells have the ability to metabolize the tetrazolium salt WST-1 into a formazan dye. The amount of formed formazan

Table 1 Concentrations for the combinatorial treatment with 5-aza-dC

\begin{tabular}{lccc}
\hline Modulator & D283-Med & DAOY & MEB-Med8a \\
\hline 5-Aza-dC & $3 \mu \mathrm{M}$ & $5 \mu \mathrm{M}$ & $5 \mu \mathrm{M}$ \\
VPA & $0.77 \mathrm{mM}$ & $0.77 \mathrm{mM}$ & $0.77 \mathrm{mM}$ \\
SAHA & $0.16 \mu \mathrm{M}$ & $0.16 \mu \mathrm{M}$ & $0.16 \mu \mathrm{M}$ \\
Abacavir & $0.11 \mathrm{mM}$ & $0.11 \mathrm{mM}$ & $0.11 \mathrm{mM}$ \\
Retinoic acid & $0.25 \mu \mathrm{M}$ & $0.25 \mu \mathrm{M}$ & $0.25 \mu \mathrm{M}$ \\
Resveratrol & $15 \mu \mathrm{M}$ & $15 \mu \mathrm{M}$ & $40 \mu \mathrm{M}$ \\
\hline
\end{tabular}

dye directly correlates with the number of viable cells. Measuring the formazan dye extinction at $450 \mathrm{~nm}$ wave length relative to medium control corresponds to the metabolic activity of the viable cells. IC 30 values were calculated by generating an exponential or linear trend using Microsoft Excel 2003 software.

\section{Clonogenic survival}

For clonogenic assays, cells were treated with/without $3 \mu \mathrm{M}$ (D283-Med) or $5 \mu \mathrm{M}$ (DAOY, MEB-Med8a) 5-aza-dC in cell culture flasks for three days. Subsequently, medium was renewed and supplemented with 5-aza-dC and $15 \mu \mathrm{M}$ (D283-Med, DAOY) or $40 \mu \mathrm{M}$ Resveratrol (MEB-Med8). After three days, cells were counted, seeded at three different cell densities in duplicates in 6-well cell culture plates, and normal medium without mediators was added. Ten to 14 days later, colonies were washed with PBS, fixed with ice-cold ethanol/acetone $(1: 1)$ for $10 \mathrm{~min}$, stained with Giemsa solution (1: 1 with distilled water) for 5 min, and washed with distilled water. Colonies with > 50 cells were counted indicating plating efficiency (PE). The ratio between PE of treated cells and PE of untreated cells represented the surviving fraction (SF) of clonogenic cells.

\section{Statistics}

Statistic analyses of were performed using the parametric, one-way, and paired Student's $t$-test with Microsoft Excel 2003 software. P-values $\leq 0.05(*)$ were considered as statistically significant and p-values $\leq 0.001\left({ }^{* * *}\right)$ as highly statistically significant.

Detailed drug interaction analyses regarding synergistic or additive effects were conducted using the Bliss independence (BI) model which is based on the noninteraction theory. The BI model compares the estimates of the combined effects calculated on the individual drug effects with those obtained from the experiment. Therefore, the following equation was used: $E_{i}=E_{A} \times E_{B}$, where $E_{i}$ is the estimated amount of metabolic activity of the theoretical combination of substance $\mathrm{A}$ and $\mathrm{B}$, and $E_{\mathrm{A}}$ and $E_{\mathrm{B}}$ are the experimental rates of metabolic activity of each drug alone. The interaction of both is described by the difference $\Delta E$ between the estimated and the observed rates of metabolic activity $\Delta E=E_{\text {estimated }}-$ $E_{\text {observed }}$ [35]. The non-parametric approach described by Prichard et al. was modified and used to calculate statistical significance of synergism. In each of the three independent experiments, the observed rates of metabolic activity were subtracted from the predicted values, and the average difference of each experiment was calculated. Statistically significant synergy was claimed when the average difference as well as its 95\% confidence interval was positive [36]. 


\section{Results and discussion}

To determine submaximal concentrations for the inhibition of the metabolic activity of MB cells, we performed incubation experiments with the single drugs. The mean drug concentration of the three examined cell lines which inhibits the metabolic activity by $30 \%$ (IC30) was chosen for combination treatments with 5 -aza-dC for three days (Table 1). The long-time reproductive cell survival of the combination showing the most promising results in metabolic inhibition was investigated and induction of DSB as a potential mechanism for cell death was evaluated.

\section{Effects on metabolic activity (WST-1 assay)}

After treatment with 5-aza-dC, we observed an enhanced reduction of metabolic activity in all cell lines treated for six days versus three days (Figure 1). As 5-aza-dC incorporation depends on cell cycle progression and proliferation frequency [10], the longer incubation period allows more 5-aza-dC to be incorporated into DNA. Surprisingly, 5-aza-dC exhibited the strongest inhibitory effect in slowly proliferating D283-Med cells, whereas DAOY cells, showing the shortest replication time, were much more resistant. Although 5-aza-dC-induced inhibition was stronger after 6 versus 3 days of treatment, leading to a total loss of metabolic activity in D283-Med and MEB-Med8a, about $20 \%$ of metabolic activity remained in DAOY cells. The relative 5-aza- $\mathrm{dC}$ resistance of DAOY cells versus MEBMed8a and D283-Med cells in mortality and cell growth arrest has already been shown by our workgroup [8]. This indicates that, beside the incubation period-dependent incorporation rate, other mechanisms, like repair efficiency or DNMT activity, are involved in 5-aza-dC-induced cytotoxicity.

VPA led to a strong dose-dependent decrease of metabolic activity in all three MB cell lines (Figure 2a). The individual VPA concentrations leading to 30\% inhibition (IC 30) were between $0.27 \mathrm{mM}$ (MEB-Med8a) and $0.9 \mathrm{mM}$ (D283-Med) after VPA treatment for three days. After combinatorial treatment with 5 -aza-dC, additive effects on the reduction of metabolic activity in two cell lines (DAOY, D283-Med) with a significant synergistic response in DAOY cells were observed. This is in accordance with data obtained from Yang et al. showing synergistic effects on inhibition of cell growth and induction of apoptosis in human leukemic cell lines [37]. In contrast, combined 5-aza-dC/VPA treatment of MEB-Med8a cells revealed a significant increase of $25 \%$ in metabolic activity compared to 5 -aza-dC monotherapy (Figure 3a). Conceivably in MEB-Med8a cells, VPA mainly induces G1 arrest by induction of $p 21$ expression [15] and, therefore, prevents cytotoxic 5-aza-dC incorporation into the DNA molecule.

Also, SAHA induced a concentration-dependent decrease of metabolic activity (Figure $2 \mathrm{~b}$ ). The IC 30 values were $60 \mathrm{nM}-260 \mathrm{nM}$ (MEB-Med8a, D283-Med). After simultaneous treatment with 5 -aza-dC, the metabolic activity of D283-Med and DAOY cells was only slightly reduced, compared to 5-aza-dC alone. Similarly to 5-aza-dC/VPA treatment response, MEB-Meb8a cells exhibited a significant enhancement of metabolic activity after combined treatment with SAHA (Figure 3b). Corresponding to these cell line-specific findings, differential results have also been published showing minor effects in colon carcinoma cells, but significantly enhanced cell death in ovarian cancer and leukemia cells after combinatorial 5-aza-dC/SAHA treatment [38-40].

Treatment of MB cells with abacavir resulted in a dosedependent reduction of metabolic activity (Figure 2c). Thereby, D283-Med revealed to be the most resistant among the examined cell lines showing an IC 30 value of $340 \mu \mathrm{M}$, whereas MEB-Med8a and DAOY cells exhibited IC 30 values of $70 \mu \mathrm{M}$ and $150 \mu \mathrm{M}$. The higher resistance is possibly due to a higher expression of human telomerase reverse transcriptase (hTERT) in D283-Med cells compared to DAOY cells $[3,24]$. Applying higher abacavir concentrations ( $350 \mu \mathrm{M}$ to $750 \mu \mathrm{M}$, treated for 24 to $96 \mathrm{~h}$ ), Rossi et al. reported that abacavir induces enhanced mortality in D283-Med cells, but differentiation and growth arrest in DAOY cells [3]. We found here that simultaneous

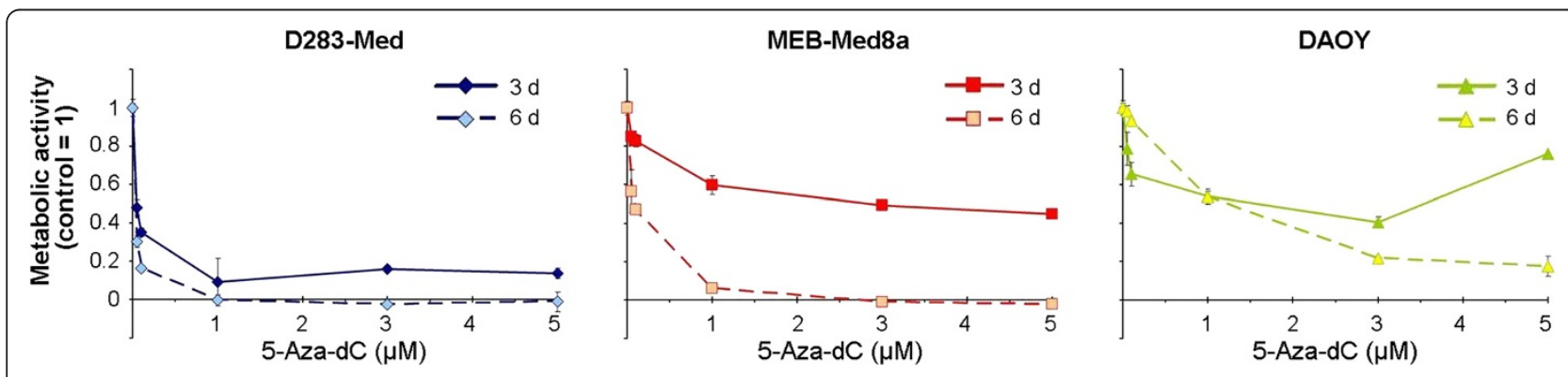

Figure 1 Time- and dose-dependent inhibition of metabolic activity by $\mathbf{5}$-aza-dC. Metabolic activity of three medulloblastoma cell lines was measured by WST-1 assay after 5-aza-dC treatment for three or six days. Raw values were normalized to untreated control. Data from one experiment are shown as means \pm SEM of triplicate samples. 


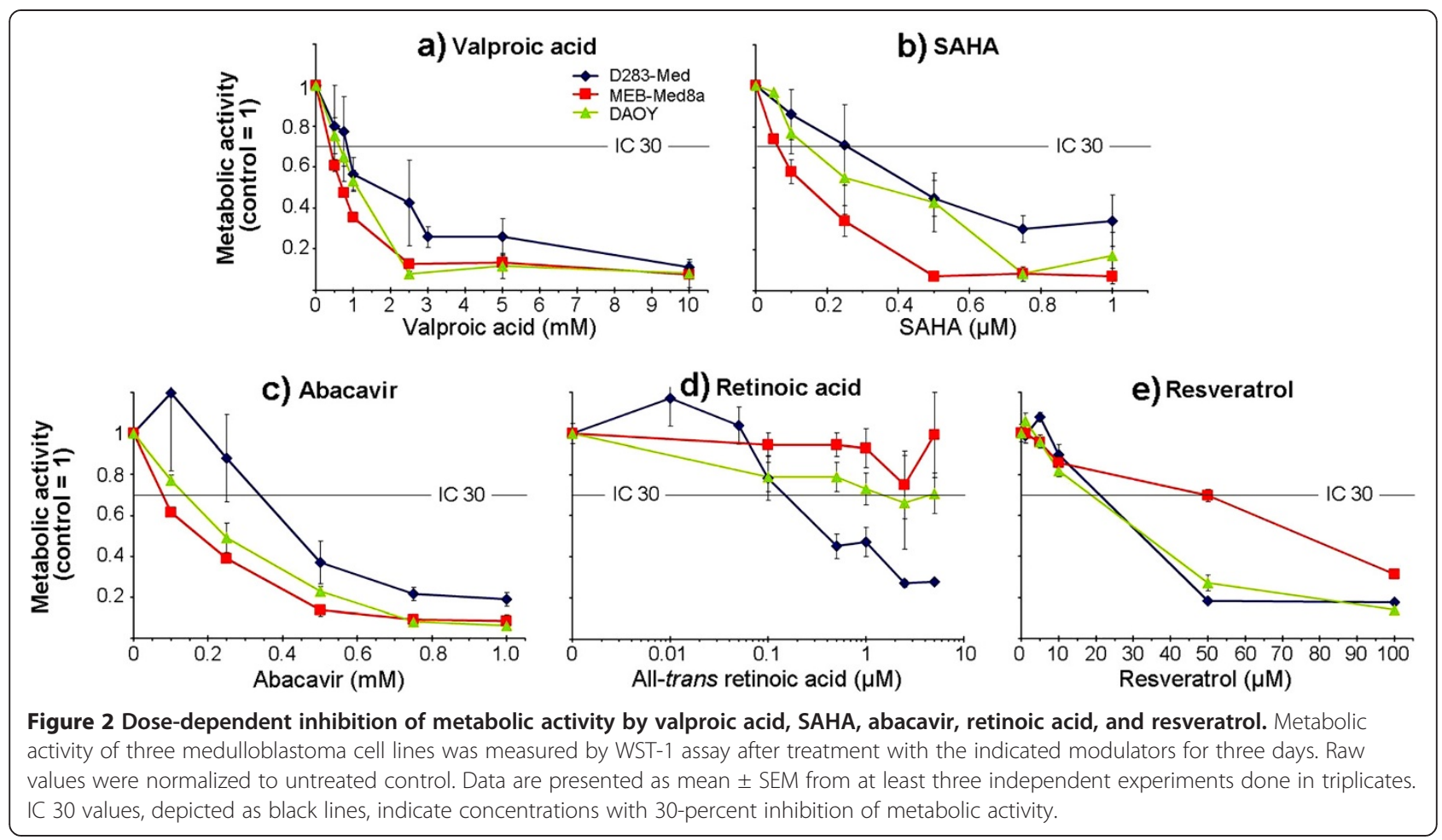

treatment with 5-aza-dC led to an additive response of two MB cell lines (DAOY, D283-Med) in metabolic activity (Figure 3c). This is the first time showing intensifying in vitro effects of an epigenetic modifier and a telomerase inhibitor on metabolic activity of tumor cells.
Retinoic acid treatment induced differential, cell linespecific effects: MEB-Med8a cells showed no response to ATRA; DAOY cells exhibited only a moderate reduction of metabolic activity with a maximum of $30 \%$; and in D283-Med cells, a dose-dependent reduction of metabolic

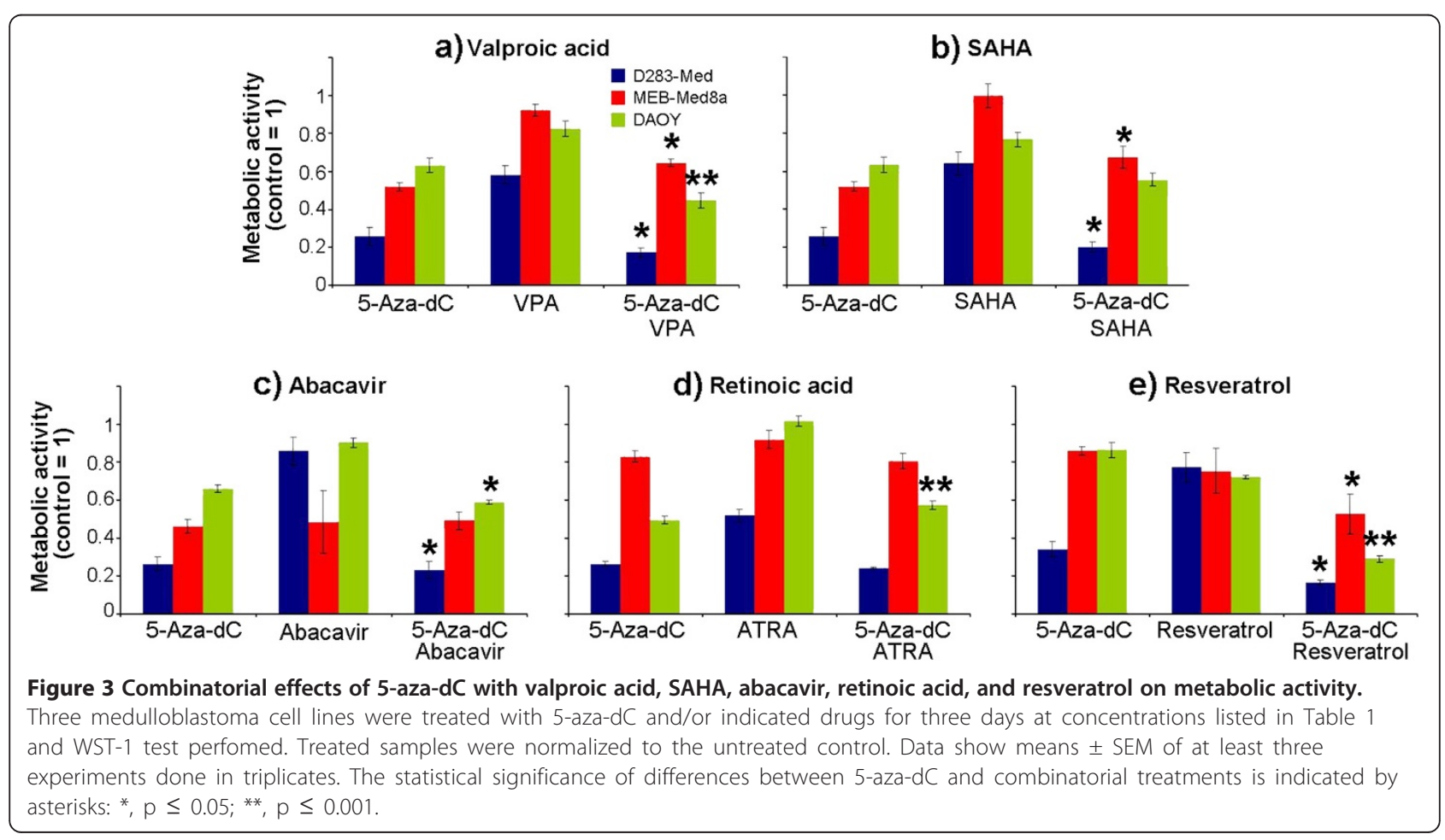




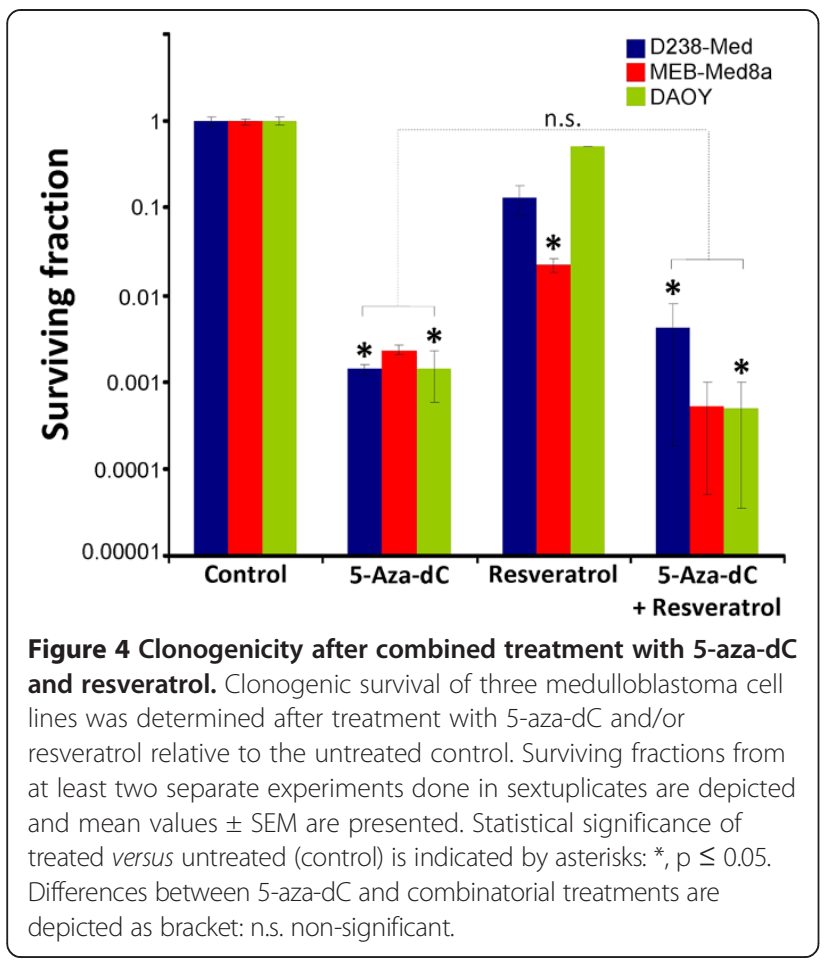

activity with up to $70 \%$ inhibition could be observed (Figure $2 \mathrm{~d}$ ). This goes along with findings of other groups $[28,30,41]$. In the highly sensitive D283-Med cell line, an ATRA-mediated caspase 3 induction followed by apoptosis has been reported [28]. In contrast, DAOY cells showed only a moderate ATRA-induced enhancement of caspase 3 expression and apoptosis [30]. The presence of OTX2 (orthodenticle homeobox 2), a homeobox protein acting as a transcription factor during brain development, seems to be necessary for ATRA-induced mortality of tumor cells. In accordance, enhanced OTX2 protein levels have been observed in the sensitive D283-Med cells, whereas the relatively resistant DAOY cells do not express OTX2 [41]. The combinatorial treatment with 5-aza-dC revealed no further effect in the ATRA-sensitive D283Med cells but led to a significant increase of metabolic activity in DAOY cells compared to 5-aza-dC alone. The simultaneous treatment of the ATRA-resistant MEBMed8a cells showed no 5-aza-dC-dependent effect on the ATRA responder status (Figure 3d). In contrast, $\mathrm{Fu}$ et al. reported a 5-aza-dC-induced hypomethylation of the hypermethylated CRABP-II (cellular retinoic acidbinding protein) gene promoter in ATRA-resistant MB cells leading to the expression of the afore-silenced gene. This affects the ATRA transport into the nucleus and lead to an ATRA-mediated cellular response in these MB cells [47]. However, the lack of an ATRA response in MEB-Med8a after combined treatment with 5-aza-dC indicates that hypermethylation of the CRABP-II promoter is not responsible for ATRA resistance in this MB cell line.

As shown in Figure 2e, resveratrol $(>10 \mu \mathrm{M})$ led to a significant concentration-dependent reduction of metabolic activity in all three examined cell lines, possibly by inhibition of STAT3 (signal transducer and activator of transcription 3) expression and activity, which results in irreversible cell cycle arrest or apoptosis [44]. The IC 30 values of $15 \mu \mathrm{M}$ (D283-Med, DAOY) and $40 \mu \mathrm{M}$ (MEBMed8a) are within the concentrations of $40 \mu \mathrm{M}$, maximal achievable in blood serum after intravenous injection [42]. The combined administration of resveratrol and 5-aza-dC showed a significant synergistic inhibition of 18\% (MEB-Med8a), 41\% (D283-Med) and 54\% (DAOY) on metabolic activity versus 5 -aza-dC alone (Figure 3e). The sensitive response of the TP53-mutated DAOY cell line might indicate a speculative role of resveratrol in the therapy of highly aggressive and therapyresistant TP53-mutated MB tumors. Numerous studies, regarding the outcome of TP53-mutated MBs, which represents about $10 \%$ of all MBs, showed a 5-year eventfree survival of $0 \%$ [43-47]. Interestingly, resveratrol has been shown to induce apoptosis p53-dependently and also p53-independently $[48,49]$.
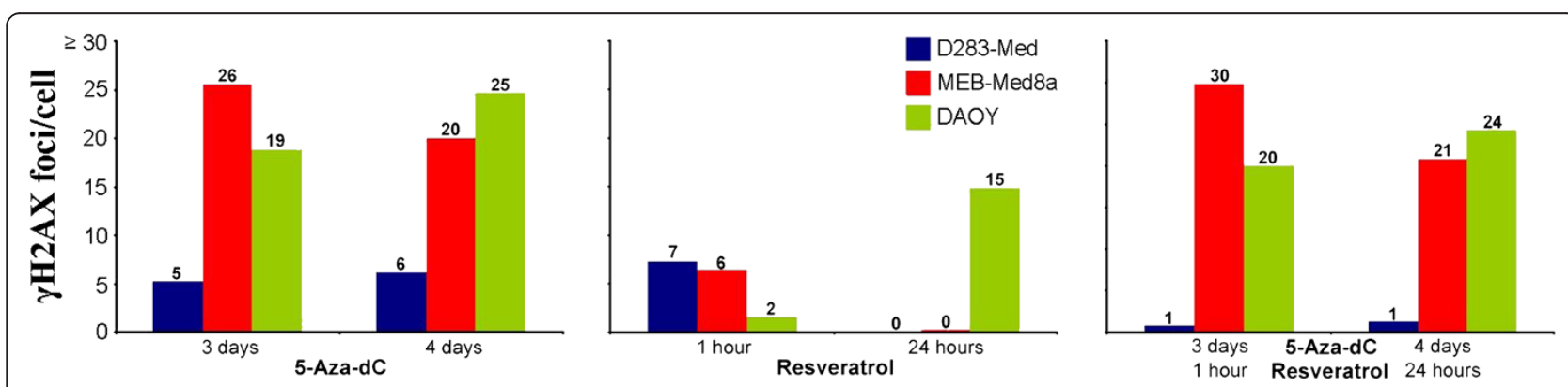

Figure 5 DSB induction after 5-aza-dC and/or resveratrol treatment. Induction of DNA double-strand break repair was measured by $\mathrm{YH} 2 \mathrm{AX}$ assay in three medulloblastoma cell lines after treatment with 5-aza-dC and/or resveratrol. Numbers of foci per cell less initial number of untreated cells are represented. One experiment was performed containing at least 50 counted cell nuclei. 


\section{Combinatorial effects of 5-aza-dC and resveratrol on clonogenicity and DSB repair}

Our investigations on metabolic activity revealed that 5-aza-dC combined with resveratrol achieve the highest antitumor response compared to the other tested drugs. To assess long-time effects, we determined the reproductive cell survival by clonogenic assay after combined 5-aza-dC and resveratrol treatment. 5-Aza-dC alone resulted in a decrease of surviving clonogenic cells exhibiting surviving fractions (SF) between 0.0014 (DAOY, D283-Med8) and 0.0023 (MEB-Med8a), similar to our previously published data [8]. After resveratrol treatment, a significant decline of clonogenic survival was only observed in MEB-Med8a leading to a SF of 0.022, whereas, in DAOY and D283-Med, only small effects were seen $\left(\mathrm{SF}_{(\mathrm{DAOY})}=0.52 ; \mathrm{SF}_{(\mathrm{D} 283-\mathrm{Med})}=0.13\right)$. The combinatorial treatment with 5 -aza-dC and resveratrol revealed no overall decline but cell line-specific effects on clonogenic survival. A resveratrol-mediated enhancement of 5-aza-dC-induced clonogenic cell death was observed in MEB-Med8a and DAOY with a reduction by $78 \%(\mathrm{SF}=0.0005)$ and $64 \%(\mathrm{SF}=0.0005)$ versus 5 -aza-dC alone. In contrast, resveratrol showed protective effects on clonogenicity of D283-Med cells represented by a 2.9 fold enhancement $(\mathrm{SF}=0.0041)$ in clonogenic survival of 5 -aza-dC-treated cells (Figure 4).

A common mechanism for the initiation of clonogenic cell death is the induction of DSB [50]. Therefore, we measured the DSB indirectly by immune fluorescence staining of $\gamma \mathrm{H} 2 \mathrm{AX}$ repair protein $1 \mathrm{~h}$ and $24 \mathrm{~h}$ after resveratrol treatment. 5 -Aza-dC or resveratrol alone caused the formation of $\gamma \mathrm{H} 2 \mathrm{AX}$ foci, although there was no correlation between initial $(1 \mathrm{~h})$ nor residual $(24 \mathrm{~h})$ foci number and surviving fraction. Palii et al. have previously described the DSB-inducing cytotoxic capabilities of 5-aza-dC in cervix and colon carcinoma cells [12]. Also, it was shown that resveratrol influences the DSB repair cascade and, thereby, induces $\gamma \mathrm{H} 2 \mathrm{AX}$ foci in ovarian cancer cells [51]. Adjuvant resveratrol administration exhibits no further effects on the 5-aza-dCinduced DSB repair, as no additional foci induction in MEB-Med8a and DAOY cells was found. Contrary to this, in D283-Med cells even a decrease of DSB formation was detected (Figure 5) which is going along with our findings showing an enhancement of clonogenic survival. Moreover, the resveratrol-mediated induction of base excision repair [52] which is shown to be p53-dependent [53], might reduce the priorly DNA-incorporated 5-aza-dC in p53 wild-type D283Med cells. Possibly, similar mechanisms are responsible for the protective effects of resveratrol on the survival of normal cells after chemotherapeutical treatment $[54,55]$.

\section{Conclusions}

Combination of 5-aza-dC and differentiation-inducing or epigenetic mediators show promising antitumor effects on metabolic activity of MB cells. Cell line-specific results indicate an important impact of the genetic background, which is known to be extremely variable in MBs. Further insight in the acting mechanisms, especially of resveratrol, is needed to evaluate the full potential in antitumor therapy and to translate the synergistic effects on short-term metabolic activity into long-term reproductive survival deficiency.

\section{Competing interests}

The authors declare no conflict of interest.

\section{Authors' contribution}

IP is participated in the design of the study, carried out the experimental assays and draft the manuscript. AG is participated in conceiving the study and helped to draft the manuscript. RK take part in research instruction and development of the manuscript. All authors read and approved the final manuscript.

Received: 25 March 2013 Accepted: 7 May 2013

Published: 14 May 2013

\section{References}

1. Gibson P, Tong Y, Robinson G, Thompson MC, Currle DS, Eden C, Kranenburg TA, Hogg T, Poppleton H, Martin J, et al: Subtypes of medulloblastoma have distinct developmental origins. Nature 2010, 468:1095-1099.

2. de Bont JM, Packer RJ, Michiels EM, den Boer ML, Pieters R: Biological background of pediatric medulloblastoma and ependymoma: a review from a translational research perspective. Neuro Oncol 2008, 10:1040-1060.

3. Rossi A, Russo G, Puca A, La MR, Caputo M, Mattioli E, Lopez M, Giordano A, Pentimalli F: The antiretroviral nucleoside analogue Abacavir reduces cell growth and promotes differentiation of human medulloblastoma cells. Int J Cancer 2009, 125:235-243.

4. Yu LJ, Wu ML, Li H, Chen XY, Wang Q, Sun Y, Kong QY, Liu J: Inhibition of STAT3 expression and signaling in resveratrol-differentiated medulloblastoma cells. Neoplasia 2008, 10:736-744.

5. Wang Q, Li H, Wang XW, Wu DC, Chen XY, Liu J: Resveratrol promotes differentiation and induces Fas-independent apoptosis of human medulloblastoma cells. Neurosci Lett 2003, 351:83-86.

6. Chai G, Li L, Zhou W, Wu L, Zhao Y, Wang D, Lu S, Yu Y, Wang H, McNutt MA, et al: HDAC inhibitors act with 5-aza-2'-deoxycytidine to inhibit cell proliferation by suppressing removal of incorporated abases in lung cancer cells. PLoS One 2008, 3:e2445.

7. Fu YS, Wang Q, Ma JX, Yang XH, Wu ML, Zhang KL, Kong QY, Chen XY, Sun $Y$, Chen NN, et al: CRABP-II methylation: a critical determinant of retinoic acid resistance of medulloblastoma cells. Mol Oncol 2012, 6:48-61.

8. Patties I, Jahns J, Hildebrandt G, Kortmann RD, Glasow A: Additive effects of 5-aza-2'-deoxycytidine and irradiation on clonogenic survival of human medulloblastoma cell lines. Strahlenther Onkol 2009, 185:331-338.

9. Hagemann S, Heil O, Lyko F, Brueckner B: Azacytidine and decitabine induce gene-specific and non-random DNA demethylation in human cancer cell lines. PLoS One 2011, 6:e17388.

10. Li LH, Olin EJ, Fraser TJ, Bhuyan BK: Phase specificity of 5-azacytidine against mammalian cells in tissue culture. Cancer Res 1970, 30:2770-2775.

11. Santi DV, Norment A, Garrett CE: Covalent bond formation between a DNA-cytosine methyltransferase and DNA containing 5-azacytosine. Proc Natl Acad Sci USA 1984, 81:6993-6997.

12. Palii SS, Van Emburgh BO, Sankpal UT, Brown KD, Robertson KD: DNA methylation inhibitor 5-Aza-2'-deoxycytidine induces reversible genomewide DNA damage that is distinctly influenced by DNA methyltransferases 1 and 3B. Mol Cell Biol 2008, 28:752-771. 
13. Lemaire M, Chabot GG, Raynal NJ, Momparler LF, Hurtubise A, Bernstein ML, Momparler RL: Importance of dose-schedule of 5-aza-2'-deoxycytidine for epigenetic therapy of cancer. BMC Cancer 2008, 8:128.

14. Boivin AJ, Momparler LF, Hurtubise A, Momparler RL: Antineoplastic action of 5-aza-2'-deoxycytidine and phenylbutyrate on human lung carcinoma cells. Anticancer Drugs 2002, 13:869-874.

15. Li XN, Shu Q, Su JM, Perlaky L, Blaney SM, Lau CC: Valproic acid induces growth arrest, apoptosis, and senescence in medulloblastomas by increasing histone hyperacetylation and regulating expression of p21Cip1, CDK4, and CMYC. Mol Cancer Ther 2005, 4:1912-1922.

16. Shu Q, Antalffy B, Su JM, Adesina A, Ou CN, Pietsch T, Blaney SM, Lau CC, Li $\mathrm{XN}$ : Valproic Acid prolongs survival time of severe combined immunodeficient mice bearing intracerebellar orthotopic medulloblastoma xenografts. Clin Cancer Res 2006, 12:4687-4694.

17. Ecke I, Petry F, Rosenberger A, Tauber S, Monkemeyer S, Hess I, Dullin C, Kimmina S, Pirngruber J, Johnsen SA, et al: Antitumor effects of a combined 5-aza-2'deoxycytidine and valproic acid treatment on rhabdomyosarcoma and medulloblastoma in Ptch mutant mice. Cancer Res 2009, 69:887-895.

18. Finnin MS, Donigian JR, Cohen A, Richon VM, Rifkind RA, Marks PA, Breslow R, Pavletich NP: Structures of a histone deacetylase homologue bound to the TSA and SAHA inhibitors. Nature 1999, 401:188-193.

19. Richon VM, Sandhoff TW, Rifkind RA, Marks PA: Histone deacetylase inhibitor selectively induces p21WAF1 expression and gene-associated histone acetylation. Proc Natl Acad Sci USA 2000, 97:10014-10019.

20. Rikiishi H, Shinohara F, Sato T, Sato Y, Suzuki M, Echigo S: Chemosensitization of oral squamous cell carcinoma cells to cisplatin by histone deacetylase inhibitor, suberoylanilide hydroxamic acid. Int J Oncol 2007, 30:1181-1188.

21. Hacker S, Karl S, Mader I, Cristofanon S, Schweitzer T, Krauss J, Rutkowski S, Debatin KM, Fulda S: Histone deacetylase inhibitors prime medulloblastoma cells for chemotherapy-induced apoptosis by enhancing p53-dependent Bax activation. Oncogene 2011, 30:2275-2281.

22. Tendian SW, Parker WB: Interaction of deoxyguanosine nucleotide analogs with human telomerase. Mol Pharmacol 2000, 57:695-699.

23. Shay JW, Bacchetti S: A survey of telomerase activity in human cancer. Eur J Cancer 1997, 33:787-791.

24. Chang Q, Pang JC, Li J, Hu L, Kong X, Ng HK: Molecular analysis of PinX1 in medulloblastomas. Int J Cancer 2004, 109:309-314.

25. Jones-Villeneuve EM, Rudnicki MA, Harris JF, McBurney MW: Retinoic acidinduced neural differentiation of embryonal carcinoma cells. Mol Cell Biol 1983, 3:2271-2279

26. Sidell N, Sarafian T, Kelly M, Tsuchida T, Haussler M: Retinoic acid-induced differentiation of human neuroblastoma: a cell variant system showing two distinct responses. Exp Cell Biol 1986, 54:287-300.

27. Webb M, Graham C, Walsh F: Neuronal differentiation of cloned human teratoma cells in response to retinoic acid in vitro. J Neuroimmunol 1986 11:67-86.

28. Gumireddy K, Sutton LN, Phillips PC, Reddy CD: All-trans-retinoic acidinduced apoptosis in human medulloblastoma: activation of caspase-3/poly (ADP-ribose) polymerase 1 pathway. Clin Cancer Res 2003, 9:4052-4059.

29. Chang Q, Chen Z, You J, McNutt MA, Zhang T, Han Z, Zhang X, Gong E, $\mathrm{Gu} J$ : All-trans-retinoic acid induces cell growth arrest in a human medulloblastoma cell line. J Neurooncol 2007, 84:263-267.

30. Hallahan AR, Pritchard II, Chandraratna RA, Ellenbogen RG, Geyer JR, Overland RP, Strand AD, Tapscott SJ, Olson JM: BMP-2 mediates retinoidinduced apoptosis in medulloblastoma cells through a paracrine effect. Nat Med 2003, 9:1033-1038.

31. Scott E, Steward WP, Gescher AJ, Brown K: Resveratrol in human cancer chemoprevention-choosing the 'right' dose. Mol Nutr Food Res 2012, 56:7-13.

32. Whitlock NC, Baek SJ: The anticancer effects of resveratrol: modulation of transcription factors. Nutr Cancer 2012, 64:493-502.

33. Muqbil I, Beck FW, Bao B, Sarkar FH, Mohammad RM, Hadi SM, Azmi AS: Old wine in a new bottle: the Warburg effect and anticancer mechanisms of resveratrol. Curr Pharm Des 2012, 18:1645-1654.

34. Zhang P, Li H, Wu ML, Chen XY, Kong QY, Wang XW, Sun Y, Wen S, Liu J: c-Myc downregulation: a critical molecular event in resveratrol-induced cell cycle arrest and apoptosis of human medulloblastoma cells. J Neurooncol 2006, 80:123-131.

35. Bliss Cl: The toxicity of poisons applied jointly1. Ann Appl Biol 1939, 26:585-615.
36. Prichard MN, Shipman C Jr: A three-dimensional model to analyze drugdrug interactions. Antiviral Res 1990, 14:181-205.

37. Yang H, Hoshino K, Sanchez-Gonzalez B, Kantarjian H, Garcia-Manero G: Antileukemia activity of the combination of 5-aza-2'-deoxycytidine with valproic acid. Leuk Res 2005, 29:739-748.

38. Yang D, Torres CM, Bardhan K, Zimmerman M, McGaha TL, Liu K: Decitabine and vorinostat cooperate to sensitize colon carcinoma cells to Fas ligand-induced apoptosis in vitro and tumor suppression in vivo. J Immunol 2012, 188:4441-4449.

39. Brodska B, Otevrelova P, Holoubek A: Decitabine-induced apoptosis is derived by Puma and Noxa induction in chronic myeloid leukemia cell line as well as in PBL and is potentiated by SAHA. Mol Cell Biochem 2011, 350:71-80.

40. Chen MY, Liao WS, Lu Z, Bornmann WG, Hennessey V, Washington MN, Rosner GL, Yu Y, Ahmed AA, Bast RC Jr: Decitabine and suberoylanilide hydroxamic acid (SAHA) inhibit growth of ovarian cancer cell lines and xenografts while inducing expression of imprinted tumor suppressor genes, apoptosis, G2/M arrest, and autophagy. Cancer 2011, 117:4424-4438.

41. Di C, Liao S, Adamson DC, Parrett TJ, Broderick DK, Shi Q, Lengauer C, Cummins JM, Velculescu VE, Fults DW, et al: Identification of OTX2 as a medulloblastoma oncogene whose product can be targeted by all-trans retinoic acid. Cancer Res 2005, 65:919-924.

42. Boocock DJ, Faust GE, Patel KR, Schinas AM, Brown VA, Ducharme MP, Booth TD, Crowell JA, Perloff M, Gescher AJ, et al: Phase I dose escalation pharmacokinetic study in healthy volunteers of resveratrol, a potential cancer chemopreventive agent. Cancer Epidemiol Biomarkers Prev 2007, 16:1246-1252.

43. Badiali M, Iolascon A, Loda M, Scheithauer BW, Basso G, Trentini GP, Giangaspero F: p53 gene mutations in medulloblastoma. Immunohistochemistry, gel shift analysis, and sequencing. Diagn Mol Pathol 1993, 2:23-28.

44. Wang W, Kumar P, Wang W, Whalley J, Schwarz M, Malone G, Haworth A, Kumar S: The mutation status of PAX3 and p53 genes in medulloblastoma. Anticancer Res 1998, 18:849-853.

45. Adesina AM, Nalbantoglu J, Cavenee WK: p53 gene mutation and mdm2 gene amplification are uncommon in medulloblastoma. Cancer Res 1994, 54:5649-5651

46. Saylors RL III, Sidransky D, Friedman HS, Bigner SH, Bigner DD, Vogelstein B, Brodeur GM: Infrequent p53 gene mutations in medulloblastomas. Cancer Res 1991, 51:4721-4723.

47. Tabori U, Baskin B, Shago M, Alon N, Taylor MD, Ray PN, Bouffet E, Malkin D, Hawkins C: Universal poor survival in children with medulloblastoma harboring somatic TP53 mutations. J Clin Oncol 2010, 28:1345-1350.

48. Huang C, Ma WY, Goranson A, Dong Z: Resveratrol suppresses cell transformation and induces apoptosis through a p53-dependent pathway. Carcinogenesis 1999, 20:237-242.

49. Gogada R, Prabhu V, Amadori M, Scott R, Hashmi S, Chandra D: Resveratrol induces p53-independent, $X$-linked inhibitor of apoptosis protein (XIAP)mediated Bax protein oligomerization on mitochondria to initiate cytochrome c release and caspase activation. J Biol Chem 2011, 286:28749-28760.

50. Radford IR: Evidence for a general relationship between the induced level of DNA double-strand breakage and cell-killing after X-irradiation of mammalian cells. Int J Radiat Biol Relat Stud Phys Chem Med 1986, 49:611-620.

51. Tyagi A, Singh RP, Agarwal C, Siriwardana S, Sclafani RA, Agarwal R: Resveratrol causes Cdc2-tyr15 phosphorylation via ATM/ATR-Chk1/2-Cdc25C pathway as a central mechanism for $\mathrm{S}$ phase arrest in human ovarian carcinoma Ovcar-3 cells. Carcinogenesis 2005, 26:1978-1987.

52. Yamamori T, DeRicco J, Naqvi A, Hoffman TA, Mattagajasingh I, Kasuno K, Jung SB, Kim CS, Irani K: SIRT1 deacetylates APE1 and regulates cellular base excision repair. Nucleic Acids Res 2010, 38:832-845.

53. Zhou J, Ahn J, Wilson SH, Prives C: A role for p53 in base excision repair. EMBO J 2001, 20:914-923.

54. Simsek G, Tokgoz SA, Vuralkan E, Caliskan M, Besalti O, Akin I: Protective effects of resveratrol on cisplatin-dependent inner-ear damage in rats. Eur Arch Otorhinolaryngol 2012, 270:1789-1793

55. Subbiah U, Raghunathan M: Chemoprotective action of resveratrol and genistein from apoptosis induced in human peripheral blood lymphocytes. J Biomol Struct Dyn 2008, 25:425-434.

doi:10.1186/1756-9966-32-27

Cite this article as: Patties et al:: Inhibitory effects of epigenetic modulators and differentiation inducers on human medulloblastoma cell lines. Journal of Experimental \& Clinical Cancer Research 2013 32:27. 